\title{
Synthesising Functional Invariants in Separation Logic
}

\author{
Ewen Maclean ${ }^{1}$, Andrew Ireland ${ }^{1}$ and Gudmund Grov ${ }^{2}$ \\ 1 Heriot-Watt University \\ Edinburgh \\ Scotland \\ ${ }^{2}$ University of Edinburgh \\ Edinburgh \\ Scotland
}

\section{The CORE system}

In 2] we introduced a system which used term synthesis to generate correct loop invariants. The CORE system extends this and is capable of automatically proving fully functional properties of programs involving pointers, by utilising existing systems to eliminate shape parts, and extracting function from the structural statements. The system is capable of synthesising correct functional invariants which allow proofs to succeed. We describe below how we define these terms.

\section{Shape, structure and function}

Consider the loop invariant expression for in-place list reversal:

$$
\text { data_lseg }(\alpha, i, n i l) * d a t a \_l s e g(\beta, j, n i l) \wedge \alpha_{0}=\operatorname{rev}(\beta)<>\alpha,
$$

where $<>$ represents concatenation. We define the following three properties:

Shape This describes purely the shape of the heap, and hence can be described purely as in Smallfoot as list $(i) * \operatorname{list}(j)$. as the list segments are null-terminated. There is no information about any data that is contained in the list, purely an indication of the inductive data structures that exist in this part of the heap, in this case linked lists.

Structural This describes the inductive structures on the heap, and gives names for the data contained within them. In this case the structural content is written data_list $(\alpha, i) *$ data_list $(\beta, j)$ in our system, as the list segments are null-terminated. When reasoning about functional properties, it is important that shape information is augmented with logical variables so that this can be extracted.

Functional This describes the pure fragment of the statement in separation logic. In this case $\alpha_{0}=\operatorname{rev}(\beta)<>\alpha$ describes the functional content, which importantly relies on the logical variables introduced between the shape and structural content.

\section{Invariant Synthesis}

We are able to take invariants such as data_lseg $(\alpha, i, n i l) * \operatorname{data\_ lseg}(\beta, j, n i l) \wedge \mathcal{F}\left(\alpha_{0}, \alpha, \beta\right)$ and synthesise correct instantiations for $\mathcal{F}$, and then automatically prove the verification conditions using IsaPlanner [1]. 


\section{References}

[1] L. Dixon and J. D. Fleuriot. IsaPlanner: A prototype proof planner in Isabelle. In Proceedings of CADE'03, volume 2741 of $L N C S$, pages 279-283, 2003.

[2] Ewen Maclean, Andrew Ireland, Lucas Dixon, and Robert Atkey. Refinement and Term Synthesis in Loop Invariant Generation. In Andrew Ireland and Laura Kovacs, editors, 2nd International Workshop on Invariant Generation (WING'09), pages 72-86, 2009. 